\title{
Diagnostic utility of central damage determination in glaucoma by magnetic resonance imaging: An observational study
}

\author{
MU LI ${ }^{1}$, MANLIN KE $^{1}$, YINWEI SONG ${ }^{1}$, KETAO MU $^{2}$, HONG ZHANG $^{1}$ and ZHIQI CHEN ${ }^{1}$ \\ Departments of ${ }^{1}$ Ophthalmology and ${ }^{2}$ Radiology, Tongji Hospital, Tongji Medical College, \\ Huazhong University of Science and Technology, Wuhan, Hubei 430030, P.R. China
}

Received September 3, 2018; Accepted November 30, 2018

DOI: $10.3892 /$ etm.2018.7134

\begin{abstract}
The aim of the present study was to investigate the utility of diffusion tensor imaging (DTI) parameters of the central visual pathway and the size of the lateral geniculate nucleus (LGN) in the diagnosis of primary open-angle glaucoma (POAG). To test this, 24 POAG patients and 24 age- and gender-matched controls underwent 3.0 Tesla magnetic resonance imaging examinations, including DTI and LGN structural imaging. Receiver operating characteristic (ROC) curves were generated and Spearman's correlation coefficients were also determined. The results indicated that regarding the discrimination of POAG patients from healthy controls, the fractional anisotropy (FA) values of the optic tract, at a cutoff of $\leq 0.412$, with an area under the ROC curve of 0.931, exhibited the highest sensitivity (79.2\%) and specificity (89.6\%). The FA values of the optic tract and the optic radiation were significantly correlated with LGN size, while the mean diffusivity values were not. In conclusion, the FA value of the optic tract may be a sensitive and reliable biomarker for glaucoma evaluation.
\end{abstract}

\section{Introduction}

Glaucoma is a leading cause of irreversible blindness worldwide (1) and retinal ganglion cell (RGC) degeneration is a major pathogenic characteristic of glaucoma $(2,3)$. Furthermore, a number of magnetic resonance imaging (MRI) studies have reported that glaucomatous damage only affects RGCs in the eyes, but also extends across the central visual pathway, then further affecting the optic tract, lateral geniculate nucleus (LGN) and the optic radiation (4-8). Using high-resolution 7.0-T MRI, Lee et al (4) identified that the LGN volumes are significantly

Correspondence to: Professor Zhiqi Chen, Department of Ophthalmology, Tongji Hospital, Tongji Medical College, Huazhong University of Science and Technology, 1095 Jiefang Road, Wuhan, Hubei 430030, P.R. China

E-mail: 66czq@163.com

Key words: diffusion tensor imaging, lateral geniculate nucleus, glaucoma, magnetic resonance imaging, receiver operating characteristic curves smaller in primary open-angle glaucoma (POAG) patients compared with those in healthy subjects. A similar MRI study by Chen et al (5) confirmed this result and further indicated that the LGN atrophy is consistent with the damage of the optic disc in POAG patients. Besides LGN atrophy, the fractional anisotropy (FA) of POAG patients also exhibited significant alterations, the degree of which was closely correlated with the disease severity, indicating that the atrophy of the visual cortex may be an important diagnostic index for POAG and may be utilized to guide its treatment (6,7). In addition, radiological evidence of neurodegeneration in the optic tract and optic radiation of POAG patients was provided, and this neurodegeneration was associated with the damage of the optic disc and the loss of visual function (8). Thus, radiological studies of POAG patients have yielded novel insight into the pathophysiology of glaucoma. Another previous study has also confirmed that modern MRI may be an effective and non-invasive tool for assessing the extent of glaucomatous progression within the central nervous system and provide complementary indicators of disease severity (9). However, it has remained elusive whether diffusion tensor imaging (DTI) parameters of cortical nerve fibers and LGN size determined by MRI may be used as sensitive and reliable indicators for distinguishing patients with glaucoma from healthy subjects. Therefore, the aim of the present study was to evaluate the diagnostic value of changes in DTI parameters of the central visual pathway and in the size of the LGN for differentiating glaucomatous eyes from healthy eyes, and to determine the sensitivity and specificity (diagnostic power) of these indicators in discriminating between severe glaucoma and mild to moderate glaucoma.

\section{Materials and methods}

Study subjects. The present study was approved by the Ethics Committee of Tongji Hospital (Tongji Medical College, Huazhong University of Science and Technology, Wuhan, China) and performed in accordance with the Declaration of Helsinki. Written informed consent was obtained from each subject prior to the start of the study.

The present study was performed at Tongji Hospital between April 2016 and March 2017. A total of 24 patients with POAG (age, 34.29 \pm 10.69 years; males/females, 19/5) and 24 age- and sex-matched non-glaucomatous healthy controls (age, 34.38 \pm 11.17 years; males/females, 19/5) were 
recruited for the present observational study. All of the study subjects underwent comprehensive ophthalmological examinations, including best corrected visual acuity, slit-lamp microscopy and indirect ophthalmoscope examination, intraocular pressure (IOP) measurement, anterior chamber angle examination using gonioscopy, visual field test (Octopus perimeter), central corneal thickness, axial length and retinal nerve fiber layer (RNFL) thickness (Heidelberg spectral domain optical coherence tomography) measurements (5-8). Subjects were included in the POAG group according to the following selection criteria: i) The subject was $\geq 18$ years of age; ii) a cup-to-disc (C/D) ratio $\geq 0.6$, or an interocular C/D ratio difference of $\geq 0.2$; iii) presence of an RNFL defect; iv) glaucomatous visual field defects corresponding to optic nerve changes and v) normal anterior chamber depth with an open angle. Patients who had undergone prior ocular surgeries, had a history of eye disease (except for POAG) or those with any neurological diseases were excluded from participation. Patients with systemic diseases were also excluded. Subjects were included in the normal group according to the following selection criteria: i) The subject was aged $\geq 18$ years; ii) normal IOP and fundus; iii) no significant RNFL defect; iv) normal visual field and v) normal anterior chamber depth with an open angle. Potential normal control subjects were excluded from participation if they had a family history of glaucoma, a history of ophthalmic or neurological disease or surgery, or any systemic disease. According to a study by Mills et al (10), the glaucomatous eyes were stratified into six stages, from 0 to 5, for each patient with POAG, after which the cumulative stage for the two eyes in each patient was calculated. Patients with a cumulative stage of $>7$ were assigned to the severe glaucoma group and all others were categorized into the mild to moderate glaucoma group.

MRI methodology. In accordance with previous studies $(5,8)$, MRI was performed within 7 days of the ophthalmological examinations. LGN structural imaging (Fig. 1) and DTI were performed using a 3.0 T General Electric MRI scanner (GE Healthcare Life Sciences, Little Chalfont, UK) with an eight-channel head coil. Three-dimensional-brain volume structural imaging was performed with the following parameters: Echo time $(\mathrm{TE})=3.5 \mathrm{msec}$; repetition time $(\mathrm{TR})=6.8$ msec; inversion time $=380 \mathrm{msec}$; slice thickness $=1 \mathrm{~mm}$ with no gap; matrix $=256 \times 256$; field of view $(F O V)=256 \times 256 \mathrm{~mm}^{2}$; and number of excitations (NEX) $=1$. For the proton density sequences, the parameters were as follows: $\mathrm{TE}=22 \mathrm{msec}$; $\mathrm{TR}=3,400 \mathrm{msec}$; slice thickness $=1.8 \mathrm{~mm}$ without any gap; matrix $=320 \times 320 ; \mathrm{FOV}=256 \times 256 \mathrm{~mm}^{2}$; and $\mathrm{NEX}=4$. LGN height and volume measurements were performed offline on an Advantage workstation (AW 4.4; GE Healthcare, Little Chalfont, UK). Single-shot echo planar diffusion-weighted imaging was performed with $a b_{\max }=800 \mathrm{sec} / \mathrm{mm}^{2}$, as well as 30 non-colinear gradient-encoding directions and one volume without diffusion gradients. Other acquisition parameters were as follows: TR=13,000 msec; ET=69 msec; matrix $=128 \times 128$ zero-filled to $256 \times 256 ; \mathrm{FOV}=24 \times 24 \mathrm{~cm}^{2}$; slice thickness $=2 \mathrm{~mm}$ with no gap; and NEX=2. Voxel-wise statistical analysis of FA volumes was performed using tract-based spatial analysis. Volume of interest (VOI) analysis was performed to investigate the associations between changes in FA and mean
Table I. Characteristics of POAG and normal subjects.

\begin{tabular}{lccc}
\hline Variable & $\begin{array}{c}\text { POAG } \\
\text { subjects }\end{array}$ & $\begin{array}{c}\text { Normal } \\
\text { subjects }\end{array}$ & P-value \\
\hline Age (years) & $34.29 \pm 10.69$ & $34.38 \pm 11.17$ & 0.979 \\
Sex (male/female) & $19 / 5$ & $19 / 5$ & 1.000 \\
AL $(\mathrm{mm})$ & $24.94 \pm 1.19$ & $24.87 \pm 0.55$ & 0.714 \\
CCT $(\mu \mathrm{m})$ & $537.50 \pm 35.35$ & $533.56 \pm 30.11$ & 0.558 \\
MD & $14.38 \pm 9.43$ & $0.84 \pm 0.68$ & $<0.001$ \\
RNFL thickness $(\mu \mathrm{m})$ & $61.89 \pm 21.71$ & $111.25 \pm 9.99$ & $<0.001$ \\
\hline
\end{tabular}

POAG, primary open-angle glaucoma; AL, axial length; CCT, central corneal thickness; MD, mean defect; RNFL, retinal nerve fiber layer.

diffusivity (MD). For this, the VOI masks were based on the voxels where differences in FA were detected. Subsequently, these VOI masks were back-projected onto the original images of each subject, and the MD was calculated.

Statistical analysis. All statistical analyses were performed using SPSS software v.19.0 (IBM Corp., Armonk, NY, USA). Values are expressed as the mean \pm standard deviation where applicable. Receiver operating characteristic (ROC) curves were constructed to assess the ability of each parameter to distinguish subjects with glaucoma from healthy controls and to differentiate between those with mild to moderate glaucoma and those with severe glaucoma. The area under the ROC curve was used to summarize the diagnostic accuracy of each parameter. Spearman's correlation analysis was used to determine the correlations between changes in DTI parameters and LGN size in the glaucoma group. $\mathrm{P}<0.05$ was considered to indicate a statistically significant difference.

\section{Results}

Subject characteristics. A total of 24 patients with POAG and 24 age- and sex-matched non-glaucomatous controls were included in the analysis. There were no significant differences in age $(34.29 \pm 10.69$ vs. $34.38 \pm 11.17$ years; $\mathrm{P}=0.979)$, sex ratio (male/female, $19 / 5$ vs. $19 / 5 ; \mathrm{P}=1.000)$, axial length $(24.94 \pm 1.19$ vs. $24.87 \pm 0.55 \mathrm{~mm} ; \mathrm{P}=0.714)$ and central corneal thickness $(537.50 \pm 35.35$ vs. $533.56 \pm 30.11 \mu \mathrm{m} ; \mathrm{P}=0.558)$ between the POAG and normal control groups. The mean defect of the visual filed in the POAG group was significantly higher than that in the normal control group $(14.38 \pm 9.43$ vs. $0.84 \pm 0.68$; $\mathrm{P}<0.001)$ and the RNFL in the POAG group was significantly thinner than that in the normal control group $(61.89 \pm 21.71 \mathrm{vs}$. 111.25 $\pm 9.99 \mu \mathrm{m} ; \mathrm{P}<0.001$; Table I).

Diagnostic value of MRI parameters. A ROC curve was generated to reveal the diagnostic accuracies of the various parameters in discriminating patients with POAG from normal control subjects. As presented in Table II, FA values of the optic tract and the LGN volume exhibited the highest sensitivity and specificity (FA values of the optic tract, at a cutoff of $\leq 0.412$, had a sensitivity of $79.2 \%$ and a specificity of $89.6 \%$; and the LGN volume, at a cutoff of $\leq 128 \mathrm{~mm}^{3}$, had a 

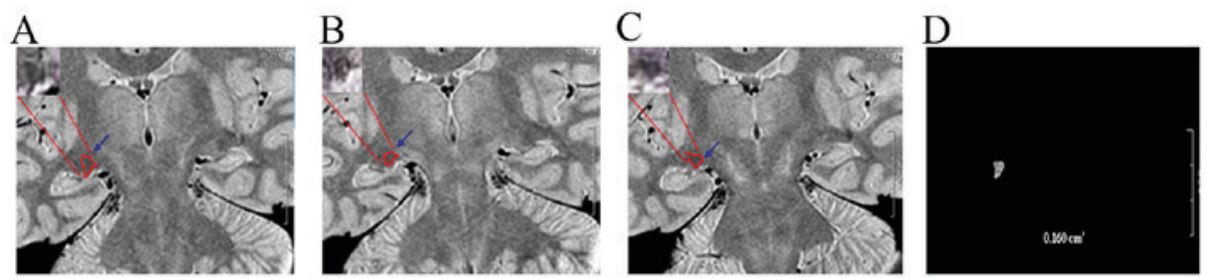

Normal control
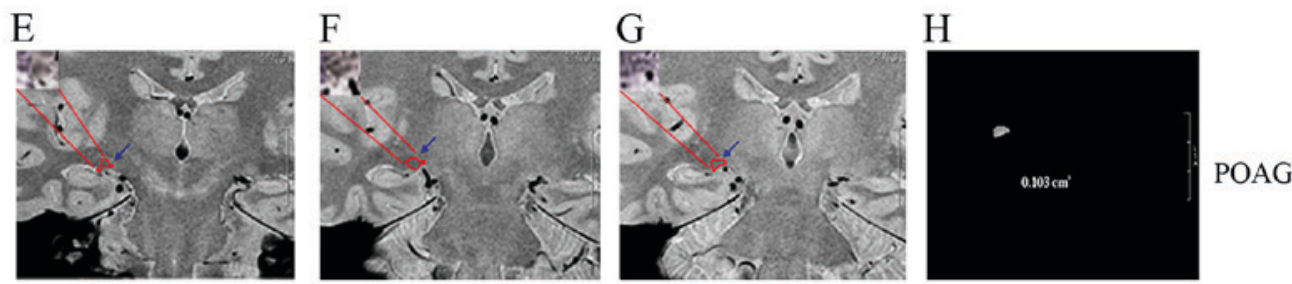

Figure 1. Determination of LGN volume from magnetic images. (A-D) Normal control and (E-H) POAG patient. First, the edge of the LGN was outlined in consecutive images on each side (areas circled with red lines indicated by blue arrows; magnification, x1.5). Subsequently, AW Volume Share 2 software was used to cut the LGN along these edges. Finally, based on the excised LGN of consecutive images, the volumes were determined. (A-C) Consecutive outlined LGN images of a normal subject (matched control) and (E-G) of a POAG patient. The volumes were determined as (D) 160 cubic millimeters for the normal control and (H) 103 cubic millimeters for the POAG patient. Full length scale bar, $3 \mathrm{~cm}$; scale bar section, $1 \mathrm{~cm}$. LGN, lateral geniculate nucleus; POAG, primary open-angle glaucoma.

sensitivity of $79.2 \%$ and a specificity of $93.7 \%$ ) for identifying patients with POAG.

The severe glaucoma group contained 12 patients in total. For discriminating between mild to moderate glaucoma and severe glaucoma, the MD (cutoff, $\leq 0.001528 \mathrm{~mm}^{2} / \mathrm{s}$; sensitivity, 87.5\%; specificity, 58.3\%) and FA (cutoff, $\leq 0.341$; sensitivity and specificity, $75.0 \%$ ) values of the optic tract exhibited the highest sensitivity and specificity (Table III).

Correlation between changes in DTI parameters and LGC size. The correlations between changes in DTI parameters and the LGN size in glaucoma are presented in Table IV. The FA values of the optic tract and the optic radiation were significantly correlated with the height $(r=0.395$ and 0.452 , respectively; $\mathrm{P}=0.005$ and 0.001 respectively) and volume $(\mathrm{r}=0.286$ and 0.348 , respectively, $\mathrm{P}=0.049$ and 0.015 , respectively) of the LGN, while the MD values were not (all $\mathrm{P}>0.05$ ).

\section{Discussion}

The earliest MRI study of structural abnormalities in glaucoma was published by Kashiwagi et al (11) in 2004. However, the majority of relevant studies has been published within the last 5 years. Most studies have determined significant anatomical degeneration within the optic nerve, optic tracts, optic chiasm, optic radiations and LGN (12-14), and these results were confirmed by a meta-analysis (15). However, few studies have focused on the diagnostic value of changes in DTI parameters of the central visual pathway and in LGN size in POAG. In a DTI study, Sidek et al (16) demonstrated that the FA of the optic nerve had a high sensitivity and specificity in distinguishing mild glaucoma from severe glaucoma. However, to date, how LGN height and volume are associated with optic fiber changes has remained elusive. Therefore, the present study aimed to provide this information in order to improve the understanding of the pathophysiological characteristics of glaucoma.

In the present study, the utility of changes in DTI parameters of the central visual pathway and in LGN size for diagnosing
POAG was evaluated. It was observed that, among the parameters determined, FA values of the optic tract had the highest sensitivity and specificity, and were reliable biomarkers for glaucoma evaluation. Furthermore, the FA values of the optic tract and optic radiation exhibited significant correlations with LGN size, providing novel insight into the central damage involved in glaucoma.

For distinguishing glaucomatous eyes from healthy eyes, measurements of the FA value of the optic tract and of the LGN volume exhibited the highest sensitivity and specificity, with cutoff values of $\leq 0.412$ and $\leq 128 \mathrm{~mm}^{3}$, respectively. Furthermore, for discriminating severe glaucoma from mild to moderate glaucoma, the MD and FA values of the optic tract exhibited the highest sensitivity and specificity. In the evaluation of the central visual pathway based on DTI parameters and LGN size, the FA of the optic tract was identified as the most reliable and useful indicator for the clinical evaluation of glaucoma. This result is in line with the results of previous studies, indicating that FA may be more sensitive to glaucomatous changes and may be an indicator of glaucomatous severity $(9,16)$. From a pathological viewpoint, these results imply that histological lesions of the optic tract in glaucoma may be more marked compared with LGN and optic radiation changes.

The correlation analysis performed to assess the link between changes in the DTI parameters and LGN size revealed that the FA values of the optic tract and optic radiation varied consistently with the LGN size, indicating that certain pathological features of central nervous system injury are associated with POAG. To understand the reasons for these correlations, it should be considered that the optic tract represents the nerve endings of the RGCs in the retina, while the optic radiation consists of the axons of LGN neurons; glaucoma is characterized by the loss of RGCs, and the loss of LGN neurons has also been indicated in pathological analyses of the brain in humans and experimental primates with glaucoma $(17,18)$. Thus, the loss of the neuron soma also leads to fiber damage, accounting for the abovementioned correlations. However, in terms of the MD values, these exhibited 
Table II. ROC curves of the LGN size and DTI parameters of the central visual pathway for discriminating between the normal $(n=24)$ and glaucoma $(n=24)$ groups.

\begin{tabular}{lccccc}
\hline Parameter & AUC & P-value & Cut-off value & Sensitivity (\%) & Specificity (\%) \\
\hline LGN height & 0.814 & $<0.001$ & $4.75 \mathrm{~mm}$ & 0.688 & 0.792 \\
LGN volume & 0.915 & $<0.001$ & $128 \mathrm{~mm}^{3}$ & 0.792 & 0.937 \\
FA-OT & 0.931 & $<0.001$ & 0.412 & 0.792 & 0.896 \\
FA-OR & 0.879 & $<0.001$ & 0.599 & 0.896 & 0.687 \\
MD-OT & 0.207 & $<0.001$ & None & None & None \\
MD-OR & 0.235 & $<0.001$ & None & None & None \\
\hline
\end{tabular}

ROC, receiver operating characteristic; AUC, area under the ROC curve; LGN, lateral geniculate nucleus; DTI, diffusion tensor imaging; FA, fractional anisotropy; MD, mean diffusivity; OT, optic tract; OR, optic radiation.

Table III. ROC curves of the LGN size and DTI parameters of the central visual pathway for distinguishing the severe glaucoma group $(n=12)$ from the mild to moderate glaucoma group $(n=12)$.

\begin{tabular}{lccccc}
\hline Parameter & AUC & P-value & Cutoff value & Sensitivity (\%) & Specificity (\%) \\
\hline LGN height & 0.682 & 0.030 & $3.95 \mathrm{~mm}$ & 0.875 & 0.417 \\
LGN volume & 0.698 & 0.019 & $67 \mathrm{~mm}^{3}$ & 0.958 & 0.208 \\
FA-OT & 0.801 & $<0.001$ & 0.341 & 0.750 & 0.750 \\
FA-OR & 0.719 & 0.009 & 0.593 & 0.583 & 0.792 \\
MD-OT & 0.802 & $<0.001$ & $0.001528 \mathrm{~mm}^{2} / \mathrm{sec}$ & 0.875 & 0.583 \\
MD-OR & 0.589 & 0.293 & None & None & None \\
\hline
\end{tabular}

ROC, receiver operating characteristic; AUC, area under the ROC curve; DTI, diffusion tensor imaging; LGN, lateral geniculate nucleus; FA, fractional anisotropy; MD, mean diffusivity; OT, optic tract; OR, optic radiation.

Table IV. Correlations between changes in DTI parameters of the central visual pathway and parameters of the LGN size in glaucoma.

\begin{tabular}{lccccc}
\hline & \multicolumn{2}{c}{ LGN height } & & \multicolumn{2}{c}{ LGN volume } \\
\cline { 2 - 3 } Parameter & rvalue & P-value & & r value & P-value \\
\hline FA-OT & 0.395 & 0.005 & & 0.286 & 0.049 \\
FA-OR & 0.452 & 0.001 & & 0.348 & 0.015 \\
MD-OT & -0.250 & 0.087 & & -0.217 & 0.139 \\
MD-OR & -0.041 & 0.781 & & -0.174 & 0.238 \\
\hline
\end{tabular}

LGN, lateral geniculate nucleus; DTI, diffusion tensor imaging; FA, fractional anisotropy; MD, mean diffusivity; OT, optic tract; OR, optic radiation.

no significant correlations. This implies that the different MR signals (FA and MD of DTI) may reflect different histological types of fiber damage $(19,20)$, and that, in the cohort of the present study, fiber damage may not have led to sufficiently marked changes in MD values. However, this hypothesis should be verified in further studies. The present results also support that in DTI studies, the FA may have the highest diagnostic value for identifying glaucoma.
LGN may serve a role in the function of perception and cognition of humans, including visual attention and awareness (21). Information on the LGN is important for understanding glaucoma-associated perception and cognition damage for future studies. The LGN scan and morphological measurements from MRI scans are simple, but the slice thickness of such a scan is $1.8 \mathrm{~mm}$, while the diameter of a normal LGN is 4-5 $\mathrm{mm}$, which may result in information dropout when examining the LGN. Furthermore, the manual delineation of the LGN may also lead to errors. Therefore, the methods used for determining the size of the LGN require further improvement. DTI is a sensitive MR technique that may detect microstructural changes in white matter (22). Compared with LGN measurements by MRI, DTI scanning is time-consuming and requires more elaborate data processing; however, it is more efficient as a diagnostic tool, particularly in terms of the measurement of FA of the optic tract. In addition, RGC axonal deficits in glaucoma appear to progress in a distal to proximal manner (23); thus, analysis of the FA value of the optic tract, which is the fiber ending of the RGCs, may facilitate an earlier diagnosis of glaucoma.

A limitation of the present study is that the study population was relatively small. This may have affected the statistical power, particularly in determining parameter sensitivity and specificity in discriminating severe glaucoma. Furthermore, older age has been significantly associated with POAG, but in the present study, most participants were under 50 years of 
age, which may have led to an age-associated bias. However, a previous MRI study (24) reported that in healthy volunteers, the FA and the MD of the bilateral optic radiation and the bilateral optic nerve had no obvious correlations with age and exhibited no obvious differences between males and females. In future studies, participants aged $>50$ years should be assessed. In addition, more biochemical experiments should be performed to verify and support the role of FA values in discriminating POAG patients from healthy individuals.

\section{Acknowledgements}

Not applicable.

\section{Funding}

No funding was received.

\section{Availability of data and materials}

The datasets used and/or analyzed during the current study are available from the corresponding author on reasonable request.

\section{Authors' contributions}

ML and YS performed the research and wrote the manuscript. $\mathrm{MK}$ and KM performed the data analysis. $\mathrm{HZ}$ and $\mathrm{ZC}$ designed the study and revised the manuscript. All of the authors read and approved the final manuscript.

\section{Ethics approval and consent to participate}

This study was approved by the Ethics Committee of Tongji Hospital, Huazhong University of Science and Technology (Wuhan, China). Informed consent was obtained from each subject prior to the start of the study.

\section{Patient consent for publication}

Patient consent for publication of the MRI images was provided.

\section{Competing interest}

The authors declare that they have no competing interests.

\section{References}

1. Flaxman SR, Bourne RRA, Resnikoff S, Ackland P,Braithwaite T, Cicinelli MV, Das A, Jonas JB, Keeffe J, Kempen JH, et al: Global causes of blindness and distance vision impairment 1990-2020: A systematic review and meta-analysis. Lancet Glob Health 5: e1221-e1234, 2017

2. Quigley HA, Dunkelberger GR and Green WR: Retinal ganglion cell atrophy correlated with automated perimetry in human eyes with glaucoma. Am J Ophthalmol 107: 453-464, 1989.

3. Urcola JH, Hernández M and Vecino E: Three experimental glaucoma models in rats: Comparison of the effects of intraocular pressure elevation on retinal ganglion cell size and death. Exp Eye Res 83: 429-437, 2006.

4. Lee JY, Jeong HJ, Lee JH, Kim YJ, Kim EY, Kim YY, Ryu T, Cho ZH and Kim YB: An investigation of lateral geniculate nucleus volume in patients with primary open-angle glaucoma using 7 tesla magnetic resonance imaging. Invest Ophthalmol Vis Sci 55: 3468-3476, 2014.
5. Chen Z, Wang J, Lin F, Dai H, Mu K and Zhang H: Correlation between lateral geniculate nucleus atrophy and damage to the optic disc in glaucoma. J Neuroradiol 40: 281-287, 2013.

6. Lu P, Shi L, Du H, Xie B, Li C, Li S, Liu T, Feng H and Wang J: Reduced white matter integrity in primary open-angle glaucoma: A DTI study using tract-based spatial statistics. J Neuroradiol 40: 89-93, 2013.

7. Tellouck L, Durieux M, Coupé P, Cougnard-Grégoire A, Tellouck J, Tourdias T, Munsch F, Garrigues A, Helmer C, Malet $\mathrm{F}$, et al: Optic radiations microstructural changes in glaucoma and association with severity: A study using 3Tesla-magnetic resonance diffusion tensor imaging. Invest Ophthalmol Vis Sci 57: 6539-6547, 2016.

8. Chen Z, Lin F, Wang J, Li Z, Dai H, Mu K, Ge J and Zhang H: Diffusion tensor magnetic resonance imaging reveals visual pathway damage that correlates with clinical severity in glaucoma. Clin Exp Ophthalmol 41: 43-49, 2013.

9. Garaci FG, Bolacchi F, Cerulli A, Melis M, Spanò A, Cedrone C, Floris R, Simonetti $G$ and Nucci C: Optic nerve and optic radiation neurodegeneration in patients with glaucoma: In vivo analysis with 3-T diffusion-tensor MR imaging. Radiology 252: 496-501, 2009.

10. Mills RP, Budenz DL, Lee PP, Noecker RJ, Walt JG, Siegartel LR, Evans SJ and Doyle JJ: Categorizing the stage of glaucoma from pre-diagnosis to end-stage disease. Am J Ophthalmol 141: 24-30, 2006.

11. Kashiwagi K, Okubo T and Tsukahara S: Association of magnetic resonance imaging of anterior optic pathway with glaucomatous visual field damage and optic disc cupping. J Glaucoma 13: 189-195, 2004.

12. Chen WW, Wang N, Cai S, Fang Z, Yu M, Wu Q, Tang L, Guo B, Feng Y, Jonas JB, et al: Structural brain abnormalities in patients with primary open-angle glaucoma: A study with 3T MR imaging. Invest Ophthalmol Vis Sci 54: 545-554, 2013.

13. Zhang YQ, Li J, Xu L, Zhang L, Wang ZC, Yang H, Chen CX, Wu XS and Jonas JB: Anterior visual pathway assessment by magnetic resonance imaging in normal-pressure glaucoma. Acta Ophthalmol 90: e295-e302, 2012.

14. Gupta N, Greenberg G, de Tilly LN, Gray B, Polemidiotis M and Yücel YH: Atrophy of the lateral geniculate nucleus in human glaucoma detected by magnetic resonance imaging. Br J Ophthalmol 93: 56-60, 2009.

15. Li K, Lu C, Huang Y, Yuan L, Zeng D and Wu K: Alteration of fractional anisotropy and mean diffusivity in glaucoma: Novel results of a meta-analysis of diffusion tensor imaging studies. PLoS One 9: e97445, 2014.

16. Sidek S, Ramli N, Rahmat K, Ramli NM, Abdulrahman F and Tan LK: Glaucoma severity affects diffusion tensor imaging (DTI) parameters of the optic nerve and optic radiation. Eur J Radiol 83: 1437-1441, 2014.

17. Gupta N, Ang LC, Noël de Tilly L, Bidaisee L and Yücel YH: Human glaucoma and neural degeneration in intracranial optic nerve, lateral geniculate nucleus, and visual cortex. Br J Ophthalmol 90: 674-678, 2006.

18. Sasaoka M, Nakamura K, Shimazawa M, Ito Y, Araie M and Hara $\mathrm{H}$ : Changes in visual fields and lateral geniculate nucleus in monkey laser-induced high intraocular pressure model. Exp Eye Res 86: 770-782, 2008.

19. Nair SR, Tan LK, Mohd Ramli N, Lim SY, Rahmat K and Mohd Nor H: A decision tree for differentiating multiple system atrophy from Parkinson's disease using 3-T MR imaging. Eur Radiol 23: 1459-1466, 2013.

20. Khong PL, Zhou LJ, Ooi GC, Chung BH, Cheung RT and Wong VC: The evaluation of Wallerian degeneration in chronic paediatric middle cerebral artery infarction using diffusion tensor MR imaging. Cerebrovasc Dis 18: 240-247, 2004.

21. Kastner S, Schneider KA and Wunderlich K: Beyond a relay nucleus: Neuroimaging views on the human LGN. Prog Brain Res 155: 125-143, 2006.

22. Basser PJ, Mattiello J and LeBihan D: Estimation of the effective self-diffusion tensor from the NMR spin echo. J Magn Reson B 103: 247-254, 1994.

23. Crish SD, Sappington RM, Inman DM, Horner PJ and Calkins DJ: Distal axonopathy with structural persistence in glaucomatous neurodegeneration. Proc Natl Acad Sci USA 107: 5196-5201, 2010.

24. Sun HH, Wang D, Zhang QJ, Bai ZL and He P: Magnetic resonance diffusion tensor imaging of optic nerve and optic radiation in healthy adults at 3T. Int J Ophthalmol 6: 868-872, 2013. 